Vol. 63 (2001) [243-255]

\title{
HELGASON SPHERES OF COMPACT SYMMETRIC SPACES AND IMMERSIONS OF FINITE TYPE
}

\author{
BANG-YEN CHEN
}

\begin{abstract}
A unit speed curve $\gamma=\gamma(s)$ in a Riemannian manifold $N$ is called a circle if there exists a unit vector field $Y(s)$ along $\gamma$ and a positive constant $k$ such that $\nabla_{s} \gamma^{\prime}(s)=k Y(s), \nabla_{s} Y(s)=-k \gamma^{\prime}(s)$. A maximal totally geodesic sphere with maximal sectional curvature in a compact irreducible symmetric space $M$ is called a Helgason sphere. A circle which lies in a Helgason sphere of a compact symmetric space is called a Helgason circle. In this article we establish some fundamental relationships between Helgason circles, Helgason spheres of irreducible symmetric spaces of compact type and the theory of immersions of finite type.
\end{abstract}

\section{INTRODUCTION}

A unit speed curve $\gamma=\gamma(s)$ in a Riemannian manifold $N$ is called a circle if there exists a vector field $Y(s)$ of unit vectors along $\gamma$ and a positive constant $k$ such that

$$
\nabla_{s} X(s)=k Y(s), \quad \nabla_{s} Y(s)=-k X(s)
$$

where $X(s)$ denotes the tangent vector of $\gamma$ and $\nabla_{s}$ the covariant differentiation along $\gamma$ at each point $\gamma(s)$. The number $1 / k$ is called the radius of the circle $\gamma$ (see [10]). A circle in an ordinary $n$-sphere $S^{n}$ is nothing but a small circle (in the usual sense) lying in a totally geodesic 2 -sphere $S^{2}$ in $S^{n}$.

Let $M$ be a Riemannian manifold. All isometries of $M$ form a Lie group which is denoted by $I(M)$. Let $G_{M}$ denote the connected component $I_{0}(M)$ of $I(M)$. The Lie group $G_{M}$ is compact if $M$ is compact (see [8]). A Riemannian manifold $M$ is called homogeneous if $G_{M}$ acts transitively on $M$. Let $o$ be a point in $M$. Denote by $K_{M}$ the isotropy subgroup at $o$. We have $M=G_{M} / K_{M}$. Very often, we simply denote $G_{M}$ and $K_{M}$ by $G$ and $K$, respectively.

A curve $\gamma$ in a homogeneous Riemannian manifold $M=G_{M} / K_{M}$ is called a homogeneous curve if it is the orbit of a point under the action of a one-parameter subgroup $\left\{\phi_{t}\right\}$ of $G=G_{M}$.

Received 15th May, 2000

Copyright Clearance Centre, Inc. Serial-fee code: 0004-9727/01 \$A2.00+0.00. 
The linear isotropy representation of $M$ is the orthogonal representation of $K$ in the tangent space $T_{o} M$ at $o$ defined by

$$
K \rightarrow O\left(T_{o} M\right): \phi \mapsto\left(\phi_{*}\right)_{o}
$$

where $\left(\phi_{*}\right)_{o}$ denotes the differential of the isometry $\phi$ at $o$ and $O\left(T_{o} M\right)$ the orthogonal group acting on the tangent space $T_{o} M$ at $o$.

A homogeneous Riemannian manifold is said to be isotropy-irreducible if its linear isotropy representation is irreducible. An isotropy-irreducible homogeneous Riemannian manifold is simply called an irreducible homogeneous Riemannian manifold.

A maximal dimensional complete totally geodesic submanifold with maximal constant curvature in a compact irreducible symmetric space $M$ is known as a Helgason sphere. Except for the case when $M$ is a real projective space the Helgason spheres are actually spheres [7]. Moreover, the dimension of every Helgason sphere in $M$ is at least two. We define a Helgason circle of $M$ to be a circle which lies in a Helgason sphere of $M$. Obviously, every isometry of $M$ carries a Helgason circle into a Helgason circle with the same radius.

The main purpose of this paper is to study the relationship between Hegalson spheres, Helgason circles and the theory of immersions of finite type. Our main results are the following.

(1) Any two Helgason circles with the same radius in an irreducible symmetric space $M$ of compact type are conjugate under the connected component $I_{0}(M)$ of the full group $I(M)$ of isometries of $M$.

(2) Every Helgason circle in an irreducible symmetric space of compact type is a homogeneous curve.

(3) An isometric immersion of class $C^{\omega}$ from an irreducible symmetric space of compact type into a Euclidean space is of finite type if and only if it carries each Helgason circle into a curve of finite type.

(4) An isometric immersion of class $C^{\omega}$ from an irreducible symmetric space of compact type into a Euclidean space is of finite type if and only if it carries each Helgason sphere into a submanifold of finite type.

\section{Curves AND Submanifolds OF Finite type}

In this section we review briefly some basic facts on submanifolds of finite type (for the details, see $[1,2,3])$.

Let $M$ be a compact Riemannian manifold. Denote by $C^{\infty}(M)$ the set of differentiable real-valued functions defined on $M$. Define an inner product on $C^{\infty}(M)$ by $\langle f, h\rangle=\int_{M} f g * 1$. This inner product turns $C^{\infty}(M)$ into a pre-Hilbert space. It is 
well-known that the eigenvalues of the Laplacian $\Delta$ form a discrete infinite sequence: $0=\bar{\lambda}_{0}<\bar{\lambda}_{1}<\bar{\lambda}_{2}<\ldots \nearrow \infty$. Let $V_{k}$ be the eigenspace of $\Delta$ associated with eigenvalue $\bar{\lambda}_{k}$. Then each $V_{k}$ is finite-dimensional and $\sum_{k=0}^{\infty} V_{k}$ is dense in $C^{\infty}(M)$ (in the $L^{2}$-sense). If we denote by $L^{2}(M)$ the completion of $\sum_{k=0}^{\infty} V_{k}$, we have $C^{\infty}(M)=L^{2}(M)$ (in the $L^{2}$-sense with respect to the topology of uniform convergence).

For a nonconstant function $f$ in $C^{\infty}(M)$, one can make the following spectral resolution (or decomposition) of $f$ (see [2] for details):

$$
f=f_{0}+\sum_{i=1}^{\infty} f_{i}, \quad f_{i} \in V_{i}
$$

The set $T(f)=\left\{i>0: f_{i} \neq 0\right\}$ is called the type of $f$. The function $f$ is said to be of finite type if $T(f)$ is a finite set; and $f$ is of infinite type if $T(f)$ is an infinite set. Moreover, $f$ is said to be of $k$-type if $T(f)$ contains exactly $k$ elements.

The upper order $(f)$ and the lower order $(f)$ of $f$ are defined by

$$
\text { upper } \operatorname{order}(f)=\sup T(f) \text { and } \quad \text { lower } \operatorname{order}(f)=\min T(f) \text {. }
$$

The same can be repeated for vector-valued differentiable functions on $M$. In particular, we can define the notions of type, order, finite type, infinite type, $k$-type, upper order and lower order for isometric immersions of a compact Riemannian manifold into a Euclidean space as well.

The simplest manifolds are those of dimension one. Let $\gamma: S^{1} \rightarrow \mathrm{E}^{m}$ be an isometric immersion of a closed smooth curve of length $2 \pi r$ into $\mathbf{E}^{m}$. Denote by $s$ the arc length of $S^{1}$.

For a periodic function $f=f(s)$ with period $2 \pi r, f(s)$ has a Fourier series expansion given by

$$
f(s)=a_{0}+\sum_{j=1}^{\infty}\left(a_{j} \cos \left(\frac{j s}{r}\right)+b_{j} \sin \left(\frac{j s}{r}\right)\right)
$$

where $a_{0}$ is a constant and $a_{j}, b_{j}$ are the Fourier coefficients of $f(s)$ given by

$$
a_{j}=\frac{1}{\pi r} \int_{-\pi r}^{\pi r} f(s) \cos \left(\frac{j s}{r}\right) d s, \quad b_{j}=\frac{1}{\pi r} \int_{-\pi r}^{\pi r} f(s) \sin \left(\frac{j s}{r}\right) d s
$$

In terms of Fourier series expansion, we have the following [2]. 
Proposition 2.1. Let $\gamma: S^{1} \rightarrow \mathrm{E}^{m}$ be a closed smooth curve of length $2 \pi r$ in $\mathbf{E}^{m}$. Then $\gamma$ is of finite type if and only if the Fourier series expansion of each coordinate function of $\gamma$ has only finitely many nonzero terms.

Proposition 2.1 can be restated as the following.

PROPOSITION 2.2. Let $\gamma: S^{1} \rightarrow \mathrm{E}^{m}$ be a closed smooth curve of length $2 \pi r$ in $\mathbf{E}^{m}$. Then $\gamma$ is of finite type if and only if each coordinate function of $\gamma$ is an algebraic polynomial in $\cos (s / r)$ and $\sin (s / r)$.

If $\gamma$ is a closed $k$-type curve $\gamma$ in $\mathrm{E}^{m}, \gamma$ can be expressed in the form:

$$
\gamma(s)=c_{0}+\sum_{i=1}^{k}\left(a_{i} \cos \left(\lambda_{t_{i}} s\right)+b_{i} \sin \left(\lambda_{t_{i}} s\right)\right), \quad \lambda_{t_{1}}<\cdots<\lambda_{t_{k}}
$$

where $a_{0}, a_{1}, \ldots, a_{k}, b_{1}, \ldots, b_{k}$ are vectors in $\mathbf{E}^{m}$ satisfying $\left|a_{i}\right|^{2}+\left|b_{i}\right|^{2} \neq 0$ for $i \epsilon$ $\{1,2, \ldots, k\}$. The type of $\gamma$ is then given by $T(\gamma)=\left\{t_{1}, \ldots, t_{k}\right\}$; moreover, the lower and upper orders of $\gamma$ are given by $t_{1}$ and $t_{k}$, respectively. A 1-type closed curve in $\mathbf{E}^{m}$ is a nothing but a circle lying in a 2-plane.

Suppose $\gamma: S^{1} \rightarrow \mathbf{E}^{m}$ is a finite type curve. By regarding $S^{1}$ as a circle in the $u v$-plane defined by $u^{2}+v^{2}=r^{2}$, each coordinate $\gamma_{A}$ of $\gamma$ is a polynomial in $u, v$ whose degree is given by the upper order of $\gamma_{A}$, for $A \in\{1, \ldots, m\}$. Clearly, the upper order of $\gamma_{A}$ is at most the upper order of $\gamma$.

\section{INVARIANT SUBSPACES AND CLASS ONE REPRESENTATIONS}

Let $M=G / K$ be a compact symmetric space, where $K$ is the isotropy subgroup at a point $o \in M$. Then $M$ is an analytic manifold and $G$ has a natural orthogonal representation $\rho$ over the Hilbert space $L^{2}(M)$ given by the action:

$$
\rho(g): L^{2}(M) \rightarrow L^{2}(M): f \mapsto f \circ g^{-1}
$$

for $g \in G$.

Every irreducible invariant subspace of $L^{2}(M)$ is finite-dimensional and it consists of analytic functions. Moreover, the space generated by the irreducible invariant subspaces of $L^{2}(M)$ is a dense subset of $L^{2}(M)$ with respect to the topology of uniform convergence. Furthermore, if $M$ is isotropy-irreducible, then every irreducible invariant subspace of $L^{2}(M)$ is contained in one of the eigenspaces $V_{i}$ of the Laplacian of $M$.

If $\widehat{G}$ is a compact Lie group and $\widehat{K}$ a closed subgroup of $\widehat{G}$, then a representation $\sigma$ of $\widehat{G}$ in a Euclidean vector space $V$ is said to be of class one with respect to $\widehat{K}$ if there exists a nonzero vector $v \in V$ which is fixed by $\widehat{K}$, that is, $\sigma(\varphi) v=v$ for every $\varphi \in \widehat{K}$. 
Denote by $\operatorname{Irr}(\widehat{G}, \widehat{K})$ a collection of mutually nonequivalent irreducible class one representations of $\widehat{G}$ with respect to $\widehat{K}$ such that every irreducible class one representation of $\widehat{G}$ with respect to $\widehat{K}$ is equivalent to one of the representations $\operatorname{in} \operatorname{Irr}(\widehat{G}, \widehat{K})$.

Let $W$ be an invariant subspace of $L^{2}(M)$ and let $\zeta_{1}, \ldots, \zeta_{m_{W}}$ be an orthonormal basis of $W$. Define a map $\psi_{W}: M \rightarrow W$ by

$$
\psi_{W}(p)=\sum_{i=1}^{m_{W}} \zeta_{i}(p) \zeta_{i} .
$$

The following results are known (see $[6,11])$.

Proposition 3.1. Let $M=G / K$ be an irreducible symmetric space of compact type. Then the orthogonal direct sum:

$$
\sum_{\sigma \in \operatorname{Irr}(G, K)} W_{\sigma}
$$

is a dense subset of $C^{\infty}(M)$ and of $L^{2}(M)$ in the $L^{2}$-sense with respect to the topology of uniform convergence, that is, every irreducible subrepresentation of $\rho$ is of class one with respect to $K$ and each irreducible class one representation appears exactly once.

Proposition 3.2. Suppose that $M=G / K$ is an irreducible symmetric space of compact type and $f \in L^{2}(M)$. Let $V(f)$ denote the closure of the vector space generated by the orbit of $f$ under $\rho(G)$ and let $f=\sum_{\sigma \in \operatorname{Irr}(G, K)} f_{\sigma}$ denote the decomposition of $f$ with respect to the invariant subspaces $W_{\sigma}$ given in Proposition 3.1. Then

$$
V(f)=\sum W_{\sigma},
$$

where the sum is taken over all $\sigma \in \operatorname{Irr}(G, K)$ with $f_{\sigma} \neq 0$.

Proposition 3.3. Let $M=G / K$ be an irreducible symmetric space of compact type and let $W$ be an invariant subspace of $L^{2}(M)$ that does not consist of constant functions only. Then

(1) $\psi_{W}$ is a $\rho_{W}$-equivariant map, where $\rho_{W}$ is the restriction of $\rho$ to $W$.

(2) $\rho_{W}$ is of class one with respect to $K$.

(3) There exists a constant $c_{W}$ such that $\widehat{\psi}_{W}=c_{W} \psi_{W}$ is an isometric immersion.

(4) If $W$ is contained in an eigenspace $V$ of the Laplacian of $M$ with eigenvalue $\lambda$, then $\widehat{\psi}_{W}$ is a minimal immersion of $M$ into a hypersphere of $W$ with radius $\sqrt{n / \lambda}$, where $c_{W}=\sqrt{n \operatorname{vol}(M) / m_{W} \lambda}, n=\operatorname{dim} M$, and $m_{W}=\operatorname{dim} W$. 


\section{Helgason Circles, Helgason spheres and finite type immersions}

The purpose of this section is to prove the following relations between Helgason circles, Helgason spheres and immersions of finite type.

THEOREM 4.1. Let $M$ be an irreducible symmetric space of compact type. Then

(1) Any two Helgason circles with the same radius are conjugate under the connected component $I_{0}(M)$ of the full group $I(M)$ of isometries of $M$.

(2) Every Helgason circle of $M$ is a homogeneous curve.

(3) Every finite type isometric immersion of $M$ into a Euclidean space carries each Helgason circle of $M$ into a curve of finite type.

(4) Every finite type isometric immersion of $M$ carries each Helgason sphere of $M$ into a submanifold of finite type.

Proof: (1) Assume that $M$ is a compact irreducible symmetric space with maximal sectional curvature $\kappa$. Observe that each Helgason circle in $M$ is contained in a 2-dimensional totally geodesic submanifold, say $S_{\gamma}^{2}$, of constant curvature $\kappa$. Let $\beta$ be another Helgason circle with the same radius. Suppose that $\beta$ lies in a 2-dimensional totally geodesic submanifold, say $S_{\beta}^{2}$, of constant curvature $\kappa$. Let $p$ and $\bar{p}$ be the points in $S_{\beta}^{2}$ and $S_{\gamma}^{2}$ so that $\beta$ and $\gamma$ are the geodesic circles in $S_{\beta}^{2}$ and $S_{\gamma}^{2}$ centred at $p$ and $\bar{p}$, respectively. Then there is an isometry $\phi \in I_{0}(M)$ which carries $S_{\beta}^{2}$ onto $S_{\gamma}^{2}[8$, Theorem 11.1, p.334]. Since $M$ is a homogeneous Riemannian manifold, we may choose $\phi$ which also carries $p$ to $\bar{p}$. Because $\beta$ and $\gamma$ have the same radius, the distance from $p$ to $\beta$ in $S_{\beta}^{2}$ and that from $\bar{p}$ to $\gamma$ in $S_{\gamma}^{2}$ are the same. Thus, $\phi$ carries the Helgason circle $\beta$ to the Helgason circle $\gamma$.

(2) Suppose $\gamma$ is a Helgason circle of a compact irreducible symmetric space $M$. Then $\gamma$ lies in a Helgason sphere $S$ of $M$. Assume $S=G_{S} / K_{S}$, where $G_{S}$ is the group of isometries on $S$ generated by point symmetries of $S$. Since each point symmetry of the totally geodesic submanifold $S$ can be extended to a point symmetry of $M, G_{S}$ can be regarded as a subgroup of $I_{0}(M)$ (see [5]). Because the Hegalson sphere $S$ is of rank one, every circle in $S$ is a homogeneous curve in $S$ (see [9]). Therefore, the Helgason circle $\gamma$ is the orbit of a point in $S$ under the action of a one-parameter subgroup of $G_{S}$. By regarding this one-parameter subgroup as a one-parameter subgroup of $I_{0}(M)$, it follows that $\gamma$ is a homogeneous curve in $M$.

(3) Let $x$ be a finite type isometric immersion of a compact irreducible symmetric space $M$ into a Euclidean space $\mathbf{E}^{m}$. We may assume that the centre of mass of $x$ in $\mathbf{E}^{m}$ is the origin of $\mathbf{E}^{m}$. Suppose that the type of $x$ is given by $T(x)=\left\{t_{1}, t_{2}, \ldots, t_{k}\right\}$. Let $\left\{\phi_{1}^{t_{i}}, \ldots, \phi_{m_{t_{i}}}^{t_{i}}\right\}$ be an orthonormal basis of the eigenspace $V_{t_{i}}$ with eigenvalue $\lambda_{t_{i}}$. 
Then the position vector of $M$ in $\mathbf{E}^{m}$ takes the form:

$$
x=\sum_{i=1}^{k}\left\{a_{1}^{i} \phi_{1}^{t_{i}}+\cdots+a_{m_{t_{i}}}^{i} \phi_{m_{t_{i}}}^{t_{i}}\right\}
$$

where $a_{1}^{1}, \ldots, a_{m_{t_{1}}}^{1}, \ldots, a_{1}^{k}, \ldots, a_{m_{t_{k}}}^{k}$ are constant vectors in $\mathbf{E}^{m}$.

Consider a standard diagonal isometric immersion $D\left(c_{t_{1}}, \ldots, c_{t_{k}}\right)$ of $M$ into $\mathbf{E}^{N}$ whose type $\left\{t_{1}, t_{2}, \ldots, t_{k}\right\}$ is the same as that of $x$. Then $D\left(c_{t_{1}}, \ldots, c_{t_{k}}\right)$ takes the form:

$$
D\left(c_{t_{1}}, \ldots, c_{t_{k}}\right)=\left(c_{t_{1}} b_{t_{1}} \phi_{1}^{t_{1}}, \ldots, c_{t_{1}} b_{t_{1}} \phi_{m_{t_{1}}}^{t_{1}}, \ldots, c_{t_{k}} b_{t_{k}} \phi_{1}^{t_{k}}, \ldots, c_{t_{k}} b_{t_{k}} \phi_{m_{t_{k}}}^{t_{k}}\right)
$$

for some positive numbers $b_{t_{1}}, \ldots, b_{t_{k}}$. Choose a standard basis $\left\{\varepsilon_{1}, \ldots, \varepsilon_{N}\right\}$ of $\mathbf{E}^{N}$. Consider the linear map $L: \mathbf{E}^{N} \rightarrow \mathbf{E}^{m}$ defined by

$$
\begin{aligned}
L\left(c_{t_{1}} b_{t_{1}} \varepsilon_{1}\right) & =a_{1}^{1}, \ldots, L\left(c_{t_{1}} b_{t_{1}} \varepsilon_{m_{t_{1}}}\right)=a_{m_{t_{1}}}^{1}, \\
\vdots & \\
L\left(c_{t_{\ell}} b_{t_{k}} \varepsilon_{m_{t_{1}}+\cdots+m_{t_{k-1}}+1}\right) & =a_{1}^{k}, \ldots, L\left(c_{t_{k}} b_{t_{k}} \varepsilon_{m_{t_{1}}+\cdots+m_{t_{k}}}\right)=a_{m_{t_{k}}}^{k}, \\
L\left(\varepsilon_{m_{t_{1}}+\cdots+m_{t_{k}}+1}\right) & =\cdots=L\left(\varepsilon_{N}\right)=0 .
\end{aligned}
$$

Then we obtain $x=L \circ D\left(c_{t_{1}}, \ldots, c_{t_{k}}\right)$. Since a standard diagonal immersion of a compact irreducible homogeneous Riemannian manifold is $G$-equivariant, the immersion $D\left(c_{t_{1}}, \ldots, c_{t_{k}}\right)$ is $G$-equivariant.

Let $\gamma$ be a Helgason circle of $M$. Then $\gamma$ is a homogeneous curve in $M$ according to statement (2). Hence the restriction of $D\left(c_{t_{1}}, \ldots, c_{t_{k}}\right)$ to $\gamma$ is equivariant. Let $\mathbf{x}$ denote the position function of the image curve $D\left(c_{t_{1}}, \ldots, c_{t_{k}}\right)(\gamma)$. Then $\mathbf{x}, \Delta_{\gamma} \mathbf{x}, \Delta_{\gamma}^{2} \mathbf{x}, \ldots, \Delta_{\gamma}^{m} \mathbf{x}$ are linearly dependent vectors fields in $\mathbf{E}^{m}$, where $\Delta_{\gamma}=-d^{2} / d s^{2}$. Therefore there exists a nontrivial polynomial $P$ of degree $m+1$ such that $P\left(\Delta_{\gamma}\right) \mathbf{x}=0$. Thus, by applying a result of [2], we conclude that the image curve is a curve of finite type. Consequently, the diagonal immersion $D\left(c_{t_{1}}, \ldots, c_{t_{k}}\right)$ carries each Helgason circle of $M$ into a curve of finite type. In particular, this shows that the Fourier series expansion of the image curve of the Helgason circle consists of only finitely many nonzero terms according to Proposition 2.1. Because the finite type isometric immersion $x$ is the composition of the standard diagonal immersion $D\left(c_{t_{1}}, \ldots, c_{t_{k}}\right)$ followed by a linear map $L$, the Fourier series expansion of $(x \circ \gamma)(s)$ also consists of only finitely many nonzero terms. Hence, the finite type immersion $x$ carries Helgason circles into curves of finite type. 
(4) Let $S$ be a Helgason sphere of a compact irreducible symmetric space $M$. The dimension of $S$ is known to be $1+m(\bar{\delta})$, where $m(\bar{\delta})$ is the multiplicity of the highest restricted root $\bar{\delta}$. Except for the case when $M$ is a real projective space, the Helgason sphere is actually a Euclidean sphere (see [7] for details). Clearly, the dimension of a Helgason sphere is at least two.

Assume that $x$ is a finite type isometric immersion of $M$ into a Euclidean space. Then $x$ carries each circle of $S$ into a curve of finite type according to statement (3). Our goal is to prove that $x$ carries the Helgason sphere $S$ into a submanifold of finite type.

First, we claim that if an isometric immersion of a 2-sphere $S^{2}$ into a Euclidean space carries each circle in $S^{2}$ into a curve of finite type, then the immersion is of finite type. Without loss of generality, we may regard $S^{2}$ as the unit sphere in Euclidean 3-space defined by $u_{0}^{2}+u_{1}^{2}+u_{2}^{2}=1$. Recall that the $i$-th nonzero eigenvalue of the Laplacian $\Delta$ on $S^{2}$ is given by $\bar{\lambda}_{i}=i(i+1)$ whose multiplicity is $m_{i}=2 i+1$. Moreover, it is known that the restriction $f$ to $S^{2}$ of a harmonic homogeneous polynomial of degree $i$ in $u_{0}, u_{1}, u_{2}$ is an eigenfunction with eigenvalue $\bar{\lambda}_{i}$. Conversely, if $f$ is an eigenfunction on $S^{2}$ with eigenvalue $\bar{\lambda}_{i}$, then there is a unique harmonic homogeneous polynomial $P$ of degree $i$ in $u_{0}, u_{1}, u_{2}$ such that $f$ is the restriction of $P$ to $S^{2}$. Let $x=\left(x_{1}, \ldots, x_{m}\right)$ denote the restriction of $x$ to $S^{2}$.

Suppose that $x$ maps $S^{2}$ into a surface of infinite type. Then there is a coordinate function $x_{A}$ whose spectral resolution contains infinitely many nonzero terms. Hence, $x_{A}$ is an infinite series in $u_{0}, u_{1}, u_{2}$ which is not an algebraic polynomial in $u_{0}, u_{1}, u_{2}$. Put

$$
x_{A}=\sum a_{i_{0} i_{1} i_{2}} u_{0}^{i_{0}} u_{1}^{i_{1}} u_{2}^{i_{2}} .
$$

Then at least one of $u_{0}, u_{1}, u_{2}$ in (4.3) has its power approach $\infty$. Without loss of generality, we may assume that the power of $u_{1}$ goes to infinity. Consider a circle $\gamma$ of $S^{2}$ with radius $r$ lying in the plane defined by $u_{0}=\sqrt{1-r^{2}}=c, 0<r<1$. We put $u_{1}=r \cos (s / r), u_{2}=r \sin (s / r)$. Substituting these equations into (4.3) yields

$$
x_{A}=\sum a_{i_{0} i_{1} i_{2}} r^{i_{1}+i_{2}} c^{i_{0}} \cos ^{i_{1}}\left(\frac{s}{r}\right) \sin ^{i_{2}}\left(\frac{s}{r}\right) .
$$

Since $\sup \left\{i_{1}: a_{i_{0} i_{1} i_{2}} \neq 0\right\}=\infty$, (4.4) cannot be an algebraic polynomial in $\cos (s / r)$ and $\sin (s / r)$. Thus, $x(\gamma)$ must be a curve of infinite type according to Proposition 2.2 , which is a contradiction. Hence, $x$ must carry each totally geodesic 2 -sphere $S^{2}$ into a surface of finite type. In particular, we have proved statement (4) if the Helgason spheres of $M$ are 2-dimensional Riemannian spheres.

Next, suppose that $M$ is not a real projective space and the dimension of the Helgason spheres is greater than 2. In this case, each Helgason sphere of $M$ is a Euclidean sphere. 
By statement (3) we know that the finite type immersion $x$ carries each Helgason circle into a curve of finite type. Thus, by applying the result we proved above, $x$ carries every circle of every totally geodesic 2-sphere $S^{2}$ of a Helgason sphere $S$ into a surface of finite type. On the other hand, it is known from [4] that an isometric immersion of compact irreducible symmetric space is of finite type if and only if it carries every geodesic into a curve of finite type. Thus, by applying this result of [4], we know that $x$ carries every geodesic of each totally geodesic 2-sphere of a Helgason sphere into a curve of finite type. Because every geodesic in a Helgason sphere $S$ is contained in some totally geodesic 2 -sphere, $x$ must carry every geodesic in a Helgason sphere into a curve of finite type. Consequently, by applying the same result of [4] again, we conclude that $x$ carries each Helgason sphere into a submanifold of finite type. This proves statement (4) except for the case when $M$ is a real projective space.

Finally, assume that $M$ is a real projective $n$-space $R P^{n}$. In this case, the Helgason sphere of $M$ is $M$ itself. Let $\pi: S^{n} \rightarrow R P^{n}$ be the canonical projection from $S^{n}$ onto $R P^{n}$. Then it is known that the $i$-th eigenspace $V_{i}$ associated with the $i$-th nonzero eigenvalue of the Laplacian on $R P^{n}$ consists of those functions $f$ for which $f \circ \pi$ belongs to the $2 i$-th eigenspace of $S^{n}$, that is, $f \circ \pi$ is the restriction to $S^{n}$ of a harmonic homogeneous polynomial in $n+1$ variables and of degree $2 i$. If $x: R P^{2} \rightarrow \mathbb{E}^{m}$ is an isometric immersion of infinite type, then there is a coordinate function $x_{A}$ of $x$ whose spectral decomposition contains infinitely many nonzero terms. Hence, if we express $x_{A}$ in the form of (4.3), then at least one of the powers of $u_{0}, u_{1}, u_{2}$ must approach $\infty$. Hence, by applying the same argument as for $S^{2}$, we know that $x$ must carry some circles in $R P^{2}$ into curves of infinite type. Consequently, if $x: R P^{2} \rightarrow \mathbb{E}^{m}$ carries every circle into a curve of finite type, then $x$ is an immersion of finite type. Therefore, by applying the cited result of [4], $x$ must carry each geodesic in $R P^{2}$ into a curve of finite type. Now, let us assume that $n>2$ and $x: R P^{n} \rightarrow \mathbb{E}^{m}$ is an isometric immersion which carries every circle in $R P^{n}$ into a curve of finite type. Then $x$ carries every circle in a totally geodesic $R P^{2}$ of $R P^{n}$ into a curve of finite type. Hence, by applying the result for $R P^{2}$, we conclude that the restriction of $x$ to the totally geodesic $R P^{2}$ is of finite type. This implies that $x$ carries every geodesic in $R P^{2}$ into a curve of finite type. Since every geodesic in $R P^{n}$ is contained in some totally geodesic $R P^{2}$, our discussion implies that $x$ carries every geodesic in $R P^{n}$ into a curve of finite type. Consequently, by applying the cited result of [4], we conclude that $x: R P^{n} \rightarrow \mathbb{E}^{m}$ is of finite type.

\section{IMMERSIONS WHICH CARRY HELGASON SPHERES INTO FINITE TYPE SUBMANIFOLDS}

The purpose of this section is to characterise immersions of compact irreducible 
symmetric spaces which carry Helgason circles and Helgason spheres into submanifolds of finite type.

THEOREM 5.1. Let $x$ be an isometric immersion of class $C^{\omega}$ from an irreducible symmetric space $M$ of compact type into a Euclidean space. Then $x$ is of finite type if and only if $x$ carries each Helgason circle of $M$ into a curve of finite type.

Proof: Assume that $x$ is an isometric immersion of class $C^{\omega}$ from an irreducible symmetric space $M$ of compact type into a Euclidean $m$-space. If $x$ is of finite type, then statement (3) of Theorem 4.1 implies that $x$ carries each Helgason circle in $M$ into a curve of finite type.

For the converse, it is sufficient to prove that every $C^{\omega}$ isometric immersion of infinite type from a compact irreducible symmetric space $M$ must carry some Helgason circles into curves of infinite type. This can be done by applying an idea of $[4,6]$. To do so it is convenient to denote the sequence of eigenvalues of the Laplacian on $M$ by $0=\lambda_{0}<\lambda_{1} \leqslant \lambda_{2} \leqslant \cdots$, where each eigenvalue is repeated according to its multiplicity. Let $\left\{\zeta_{i}\right\}_{i \in \mathbb{N}}$ denote an orthonormal basis for $L^{2}(M)$ such that $\Delta \zeta_{i}=\lambda_{i} \zeta_{i}$ for $i \in \mathbf{N}=\{0,1,2, \ldots\}$.

Define the map $\psi: M \rightarrow L^{2}(M)$ by

$$
\psi(p)=\sum_{i=1}^{\infty} \frac{\zeta_{i}(p)}{\lambda_{i}^{2 n}} \zeta_{i}, \quad n=\operatorname{dim} M
$$

The map $\psi$ is known to be well-defined, continuous and $\rho$-equivariant, where $\rho$ is the natural orthogonal representation of $G$ on $L^{2}(M)$ defined by (3.1).

Assume that $x$ is an infinite type isometric immersion of class $C^{\omega}$ from a compact irreducible symmetric space $M=G / K$ into a Euclidean $m$-space. Then at least one of its coordinate functions, say $x_{j}$, is of infinite type. We simply denote $x_{j}$ by $y$. Let

$$
y=y_{0}+\sum_{i=1}^{\infty} y_{i} \zeta_{i}
$$

be the spectral resolution of $y$. Without loss of generality, we may assume $y_{0}=0$.

Define the continuous linear map $F_{y}: L^{2}(M) \rightarrow \mathbf{R}$ by

$$
F_{y}(h)=\left\langle\Delta^{2 n} y, h\right\rangle, \quad h \in L^{2}(M)
$$

Then we have

$$
\left(F_{y} \circ \psi\right)(p)=F_{y}\left(\sum_{i=1}^{\infty} \frac{\zeta_{i}(p)}{\lambda_{i}^{2 n}} \zeta_{i}\right)=\left\langle\sum_{i=1}^{\infty} y_{i} \lambda_{i}^{2 n} \zeta_{i}, \sum_{i=1}^{\infty} \frac{\zeta_{i}(p)}{\lambda_{i}^{2 n}} \zeta_{i}\right\rangle=\sum_{i=1}^{\infty} y_{i} \zeta_{i}(p)
$$


for any $p \in M$. Therefore one has

$$
y=F_{y} \circ \psi
$$

Let $\gamma$ be a Helgason circle with length $2 \pi r$ in $M$. Then, for any $\varphi \in G$, the image $\varphi \circ \gamma$ of $\gamma$ under $\varphi$ is also a Helgason circle with length $2 \pi r$. For each $i \in \mathbf{N}$, we define two functions $a_{i}, b_{i}: G \rightarrow \mathbf{R}$ by

$$
\begin{aligned}
& a_{i}(\varphi)=\int_{-\pi r}^{\pi r}(y \circ \varphi \circ \gamma)(s) \cos \left(\frac{i s}{r}\right) d s \\
& b_{i}(\varphi)=\int_{-\pi r}^{\pi r}(y \circ \varphi \circ \gamma)(s) \sin \left(\frac{i s}{r}\right) d s
\end{aligned}
$$

which give the Fourier coefficients of the function $y \circ \varphi \circ \gamma$, for each $\varphi \in G$.

Let $L^{2}(M)=\sum_{\sigma} W_{\sigma}$ be the decomposition of $L^{2}(M)$ into irreducible invariant subspaces given in Proposition 3.1. Choose $\left\{\zeta_{i}\right\}_{i \in \mathbf{N}}$ to be an orthonormal basis adapted to this decomposition. Since the coordinate function $y$ is a function of infinite type, we may write the type $T(y)$ of $y$ in the form: $T(y)=\left\{j_{i}: i=1,2, \ldots\right\}$ so that $j_{i}$ increases with $i$. For each $i \in \mathbf{N}$, it is possible to choose $\sigma_{i} \in \operatorname{Irr}(G, K)$ such that $W_{\sigma_{i}}$ is contained in $V_{j_{i}}$ and such that $W_{\sigma_{i}}$ is not contained in the kernel of $F_{y}$. We let $n_{i}$ be the upper order of $\widehat{\psi}_{W_{\sigma_{i}}} \circ \gamma$. (See Proposition 3.3 for the definition of $\widehat{\psi}_{W_{\sigma_{i}}}$.) Then $\lim _{i \rightarrow \infty} n_{i}=\infty$. We may assume that $n_{i}$ is increasing with $i$ (by restricting to a subsequence of $\left\{j_{i}\right\}$ if necessary).

Define

$$
G_{i}=\left\{\varphi \in G: a_{n_{i}}(\varphi) \neq 0 \text { or } b_{n_{i}}(\varphi) \neq 0\right\}
$$

Then $G_{i}$ is a dense open subset of $G$ which can be seen as follows:

Let

$$
(\psi \circ \gamma)(s)=\bar{a}_{0}+\sum_{i=1}^{\infty}\left(\bar{a}_{i} \cos \left(\frac{i s}{r}\right)+\bar{b}_{i} \sin \left(\frac{i s}{r}\right)\right)
$$

be the Fourier series expansion of $(\varphi \circ \gamma)(s)$. Then, by (5.4) and (5.7) and the fact that $\psi$ is $\rho$-equivariant, we have

$$
\begin{aligned}
(y \circ \varphi \circ \gamma)(s) & =\left(F_{y} \circ \psi \circ \varphi \circ \gamma\right)(s) \\
& =\left(F_{y} \circ \rho(\varphi) \circ \psi \circ \gamma\right)(s) \\
& =F_{y}\left(\rho(\varphi) \bar{a}_{0}\right)+\sum_{i=1}^{\infty}\left(F_{y}\left(\rho(\varphi) \bar{a}_{i}\right) \cos \left(\frac{i s}{r}\right)+F_{y}\left(\rho(\varphi) \bar{b}_{i}\right) \sin \left(\frac{i s}{r}\right)\right) .
\end{aligned}
$$


Thus we find

$$
a_{i}(\varphi)=F_{y}\left(\rho(\varphi) \bar{a}_{i}\right), \quad b_{i}(\varphi)=F_{y}\left(\rho(\varphi) \bar{b}_{i}\right) .
$$

From the definition of $\sigma_{i}$ and $n_{i}$, we know that either $\bar{a}_{n_{i}}$ or $\bar{b}_{n_{i}}$ has a component in $W_{\sigma_{i}}$. Assume $\bar{a}_{n_{i}}$ has a component in $W_{\sigma_{i}}$. Then, $W_{\sigma_{i}}$ is contained in $V\left(\bar{a}_{n_{i}}\right)$.

If both $a_{n_{i}}$ and $b_{n_{i}}$ vanish on $G$, then $V\left(\bar{a}_{n_{i}}\right)$ is contained in the kernel of $F_{y}$, which is a contradiction. Thus, at least one of $a_{n_{i}}, b_{n_{i}}$ is nonzero, which implies that $G_{i}$ is an open subset of $G$. Because the immersion $x$ is of class $C^{\omega}$, the coordinate function $y$ is an analytic function. Thus, each $G_{i}$ is a dense open subset of $G$. Therefore, $\bigcap\left\{G_{i}\right\}_{i}$ is a nonempty set by Baire's theorem. We conclude that, for each element $\varphi \in \bigcap\left\{G_{i}\right\}_{i}, y \circ \varphi \circ \gamma$ is a function of infinite type. Hence the curve $x \circ \varphi \circ \gamma$ is a curve of infinite type. Therefore the immersion $x$ must carry some Helgason circles to curves of infinite type. Consequently, if the immersion $x$ carries every Helgason circle of $M$ into a curve of finite type, then $x$ is an immersion of finite type.

By applying Theorem 4.1 and Theorem 5.1 we have the following.

THEOREM 5.2. Let $x$ be an isometric immersion of class $C^{\omega}$ from an irreducible symmetric space $M$ of compact type into a Euclidean space. Then $x$ is of finite type if and only if $x$ carries each Helgason sphere of $M$ into a submanifold of finite type.

ProOF: Assume that $x$ is an isometric immersion of class $C^{\omega}$ from an irreducible symmetric space $M$ of compact type into a Euclidean space. If $x$ is of finite type, then statement (4) of Theorem 4.1 implies that $x$ carries each Helgason sphere in $M$ into a submanifold of finite type.

Conversely, assume that $x$ is a $C^{\omega}$ isometric immersion which carries every Helgason sphere in $M$ into a submanifold of finite type. Then the restriction of $x$ to each Helgason sphere is of finite type. Since each Helgason circle $\gamma$ lies in a Helgason sphere and every circle in a Helgason sphere is a homogeneous curve, the immersion $x$ must carry each Helgason circe into a curve of finite type according to Theorem 4.1. Consequently, the immersion $x$ carries each Helgason circle in $M$ into a curve of finite type. Therefore, by applying Theorem 5.1, we know that the immersion $x$ is of finite type.

\section{REFERENCES}

[1] C. Baikoussis, F. Defever, T. Koufogiorgos and L. Verstraelen, 'Finite type immersions of flat tori into Euclidean spaces', Proc. Edinburgh Math. Soc. (2) 38 (1995), 413-420.

[2] B.-Y. Chen, Total mean curvature and submanifolds of finite type, Series in Pure Mathematics 1 (World Scientific, Singapore, 1984).

[3] B.-Y. Chen, 'A report of submanifolds of finite type', Soochow J. Math. 22 (1996), 117-337. 
[4] B.-Y. Chen, J. Deprez and P. Verheyen, 'Immersions, dans un espace euclidien, d'un espace symétrique compact de rang un à géodésiques simples', C. R. Acad. Sci. Paris Sér. I Math. 304 (1987), 567-570.

[5] B.-Y. Chen and T. Nagano, 'Totally geodesic submanifolds of symmetric spaces', Duke Math. J. 44 (1977), 745-755; II ibid 45 (1978), 405-425.

[6] J. Deprez, Immersions of finite type of compact homogeneous Riemannian manifolds, Doctoral Thesis (Katholieke Universiteit Leuven, 1988).

[7] S. Helgason, 'Totally geodesic spheres in compact symmetric spaces', Math. Ann. 165 (1966), 309-317.

[8] S. Helgason, Lie groups, differential geometry and symmetric spaces, Pure and Applied Mathematics 80 (Academic Press, New York, London, 1978).

[9] K. Mashimo and K. Tojo, 'Circles in Riemannian symmetric spaces', Kodai Math. J. 22 (1999), 1-14.

[10] K. Nomizu and K. Yano, 'On circles and spheres in Riemannian geometry', Math. Ann. 210 (1974), 163-170.

[11] N. R. Wallach, 'Minimal immersions of symmetric spaces into spheres', in Symmetric space, Pure and Applied Mathematics (Marcel Dekker, New York, 1972), pp. 1-40.

Department of Mathematics

Michigan State University

East Lansing, MI 48824-1027

United States of America

e-mail: bychen@math.msu.edu 